\title{
Same-different discrepancy in an exhaustive matching task
}

\author{
MARIA JOSÉ SOLER and SALVADOR ALGARABEL \\ University of Valencia, Valencia, Spain
}

(Douglas L. Nelson, Sponsor)

\begin{abstract}
In this study, we investigated in a multistimulus matching task the size of the discrepancy between response times for "same" and response times for "different" judgments. Frequently, results have shown that "same" judgments are faster than "different" judgments. Krueger (1984) found inversion in the speed advantage when stimuli were presented simultaneously and concluded that a self-termination factor would explain this result. In the experiment reported here, the subject had to exhaustively scan the whole set of items in the stimulus string. The analysis shows no significant interaction of presentation and response type; that is, the advantage for same stimuli is not reduced for simultaneous over subsequent presentation of pairs. Neither the method of presentation nor the type of stimulus reversed the advantage of "same" judgments; however, the discrepancy between response times for "same" and "different" judgments increased with increases in set size when the number of common elements between presentation and test sets were greater.
\end{abstract}

When a subject is asked to judge whether two stimuli, normally letters, are the same or different, "same", responses are typically faster than "different" responses (the same-different discrepancy). This advantage for "same" responses has been considered counterintuitive because, if individual stimuli were analyzed on a featureby-feature basis, "different" judgments should be faster due to the possibility of the subject's self-terminating the search of the stimulus features (Sternberg, 1975). The fact that the opposite result is found in experiments is inconsistent with the self-termination assumption.

Proctor (1981) reinterpreted several findings related to the same-different discrepancy and formulated a unified theory for matching-task phenomena. According to Proctor, three processes contribute to same-different disparities: the level of processing at which the match is performed, a facilitating perceptual factor due to stimulus repetition, and an inhibitory factor involved when matching of different stimuli is implicated. Focusing on the effect of presentation type, Proctor and others (e.g., Reed, 1973) proposed that sequential presentation should favor holistic processing and that simultaneous presentation should favor analytical comparison.

Krueger (1983) found that increasing actual letter size does not alter the "same" judgment advantage of sequential over simultaneous presentation. If the increase in letter size favored an analytical type of processing, then this factor would reduce or reverse the speed advantage for "same" responses because of the self-termination factor. Moreover, the reduction or inversion of the speed advan-

Requests for reprints should be sent to Salvador Algarabel, Facultad de Psicología, Universidad de Valencia, Blasco Ibañez, 21, 46010 Valencia, Spain. tage at longer string lengths should be greater for simultaneous presentation because the presence of both letter sets would favor more analytical processing. Krueger (1983) found no effect of letter size with single matches in either simultaneous or sequential presentations. However, further research (Krueger, 1984) showed that multiletter comparisons varying in only one letter showed the fast same effect for short string length (1-3 letters) and a fast different effect for longer string lengths (5-6 letters), reversing the usual finding (see Silverman, 1974). The speed advantage for different trials was found only when stimuli were presented in a continuous manner and were always available for subjects to carry out the comparisons. When the presentation procedure was discrete, that is, when the first stimulus set disappeared with the presentation of the second, the difference disappeared.

Krueger's results show the action of a self-termination factor in the greater discrepancy of the reaction times in sequential versus simultaneous presentation. Krueger also found an indicator of self-termination in that the standard deviations of reaction times were larger for different than for same trials at all lengths "owing to the greater range of stopping points" in "different" responses (Krueger, 1984 , p. 272). In reviewing, the self-terminating principle predicts that the discrepancy between "same" and "different" judgments will be reduced with stimuli presented simultaneously.

The purpose of the research reported here was to investigate the size of the same-different discrepancy for simultaneous and sequential presentations in a modified multistimulus matching task in which the subject had to exhaustively scan the whole set of items in the stimulus set. According to Krueger (1984), sequential presentation primes the coding of the second stimulus, facilitat- 
ing the "same" response. With simultaneous presentations, at least with multiletter displays, there exists the possibility of self-termination in the case of "different" responses, which would reduce the size of the discrepancy between "same" and "different" response times.

In the present experiment, the subject saw a varying set $(1,2,3$, or 4 items) of stimuli (words or letters), defined as the comparison set, and another set of the same size called the test set. The test set was presented with or after the comparison set. The subject's task was to select the number of common elements in both sets, defined as the elements physically identical and appearing in the same positions. This restriction ensured that processing was exhaustive because the subject had to scan the whole set item by item in order to specify the number of common elements.

We expected the present experiment to shed light on the role of self-termination in multistimulus comparison. If self-termination is an important factor in the comparison process, then the same-different discrepancy should be reduced because the task used here requires an exhaustive search of the test set. The task used here differs from Krueger's (1984) task in that ours required the subject to report the number of common elements between two stimulus sets, instead of simply making a "same""different" judgment, and we used a discrete elementby-element presentation of the memory set for greater control on the subject attention mechanism. Both differences, made mainly for experimental control of the search strategy used by the subject, allow nevertheless a crosscomparison of both series of data.

\section{METHOD}

\section{Subjects}

The subjects were 56 volunteer undergraduate students ( 40 females and 16 males) at the University of Valencia. They had normal or corrected-to-normal vision.

\footnotetext{
Materials and Design

The stimuli were words and consonant letters. The words were randomly chosen from the University of Valencia wordpool (Algarabel \& Sanmartin, 1985). The words were common nouns selected from Juilland and Chang-Rodriguez's (1964) Frequency Dictionary of Spanish Words, and had between four and seven letters. The letters were consonants from the Spanish alphabet, excluding " $\tilde{n}$ " and "ll."

The subject's task was to identify the number of common elements between a sequentially or simultaneously presented series of stimuli (comparison set) and a test set. The number of items in each test or comparison set was determined by a computer program with the following restrictions: There were four blocks of trials arranged according to block size $(1,2,3$, or 4 elements), with 60 trials for each block (except for block size 4 , which had 63 trials to equate approximately the number of repeated items at each position). Within a block, every comparison and test set included a random sample of words or letters in which every match appeared the same number of times according to position in both sets. The sequences were constructed in such a way that no stimulus repetition was allowed within a trial.

In addition to the experimental blocks, the subjects received a sequence of 43 practice trials to familiarize themselves with the task. These data were discarded from the analysis.
}

\section{Procedure}

The subjects were run individually. An Apple IIe microcomputer randomized a different sequence for each subject and timed the responses. A block started with the presentation of a message with information about the size of the stimulus set of the block. After the return key was pressed, the experiment began. A trial of sequential presentation was composed of the following events: First, a comparison set was presented sequentially (stimulus by stimulus). Each stimulus was displayed for $1 \mathrm{sec}$, with a lapse of $0.5 \mathrm{sec}$ between elements of the memory set. Second, after $2 \mathrm{sec}$, the test set elements appeared simultaneously on the monitor screen and stayed there until the subject pressed a key on the computer keyboard that indicated the number of elements common to both sets (0-1 for block 1 ; 0-2 for block $2 ; 0-3$ for block 3;0-4 for block 4); for each block, the order of presentation of trials was randomized. A trial of simultaneous presentation was composed of the following events: Both the comparison and the test set were presented simultaneously, one above the other, and remained on the screen until the subject responded. The response initiated a 2 -sec intertrial interval during which no stimuli appeared on the screen. The computer provided response feedback and printed the reaction time after the subject's response.

The instructions given to the subject emphasized accuracy and speed.

\section{RESULTS}

Mean reaction times, excluding errors, for conditions in which the comparison and test sets were either the same or different were submitted to 2 (presentation type) $\times 2$ (stimulus type) $\times 4$ (set size) $\times 2$ (response type) analysis of variance, with the last two factors within subjects. "Same"-"different" responses were assimilated to responses where all stimuli were the same or different at each set size (response type). The analysis revealed that mean reaction time for all "same" responses was faster than for "different" responses $[F(1,52)=229.932$, $p<.001, M S e=1.738$ ] (see Table 1). The discrepancy between response times to "same" and "different" judgments increased as the set size increased, leading to a significant interaction of response type $\times$ set size $[F(3,156)$ $=39.266, p<.001, M S \mathrm{e}=.928]$. The effect of presen-

Table 1

Mean Response Time (RT, in msec) and Percentage of Error (PE) by Response Type, Type of Presentation, and Stimulus Type

\begin{tabular}{|c|c|c|c|c|c|c|c|c|}
\hline & \multicolumn{8}{|c|}{ Stimulus Set Size } \\
\hline & \multicolumn{2}{|c|}{1} & \multicolumn{2}{|c|}{2} & \multicolumn{2}{|c|}{3} & \multicolumn{2}{|c|}{4} \\
\hline & RT & $\mathrm{PE}$ & RT & PE & RT & PE & RT & PE \\
\hline \multicolumn{9}{|c|}{ Letters } \\
\hline \multicolumn{9}{|l|}{ Sequential } \\
\hline Same & 568 & 7.10 & 767 & 3.60 & 1027 & 4.80 & 1261 & 12.50 \\
\hline Different & 631 & 4.30 & 938 & 1.40 & 1290 & 4.80 & 1707 & 9.50 \\
\hline \multicolumn{9}{|l|}{ Simultaneous } \\
\hline Same & 753 & 1.90 & 1037 & 1.80 & 1386 & 1.90 & 1703 & 3.00 \\
\hline Different & 788 & 0.70 & 1183 & 1.10 & 1590 & 1.40 & 1812 & 0.60 \\
\hline \multicolumn{9}{|c|}{ Words } \\
\hline \multicolumn{9}{|l|}{ Sequential } \\
\hline Same & 609 & 3.30 & 858 & 2.50 & 1093 & 1.40 & 1399 & 4.80 \\
\hline Different & 691 & 2.40 & 956 & 0.40 & 1317 & 0.50 & 1698 & 5.40 \\
\hline \multicolumn{9}{|l|}{ Simultaneous } \\
\hline Same & 800 & 1.90 & 1303 & 1.10 & 1727 & 2.90 & 2129 & 2.40 \\
\hline Different & 839 & 0.70 & 1479 & 0.70 & 1959 & 3.30 & 2562 & 6.50 \\
\hline
\end{tabular}


tation type was significant $[F(1,52)=44.257, p<.001$, $M S e=38.504]$. Simultaneous presentation was slower than sequential. Presentation type interacted significantly with stimulus type $[F(1,52)=5.067, p<.05]$ and with set size $[F(3,156)=23.670, p<.001, M S \mathrm{e}=3.102]$. Mean reaction time for word sets was slower than for letter sets $[F(1,52)=10.066, p<.005, M S e=38.504]$. The discrepancy between reaction times for letters and those for words is more evident with larger sets $[F(3,156)$ $=11.731, p<.001, \mathrm{MSe}=3.102]$. Moreover, as set size increased, reaction time was greater $[F(3,156)=$ 771.258, $p<.001, M S \mathrm{e}=3.102]$.

Error rates for same and different conditions were submitted to 2 (presentation type) $\times 2$ (stimulus type) $\times 4$ (set size) $\times 2$ (response type) analysis of variance. The errors were significantly higher on sequential pairs than on simultaneous $[F(1,52)=9.833, p<.005, M S e=$ .006]. The interaction of presentation type $\times$ stimulus type was significant $[F(1,52)=8.667, p=.005$, $M S e=.006]$, as was presentation type $\times$ set size $[F(3,156)=4.000, p<.01, M S \mathrm{e}=.003]$. Finally, error rates increased with larger sets $[F(3,156)=11.000$, $p<.001, M S \mathrm{e}=.003]$.

In addition, we carried out a linear regression analysis with same sets, and another with different. The slopes of the regression line were submitted to a 2 (presentation type) $\times 2$ (stimulus type) $\times 2$ (response type) analysis of variance. This analysis shows similar results. "Different" response times were slower than "same" response times $[F(1,52)=77.296, p<.001, M S e=2825.044]$. The effect of stimulus type was significant; word comparisons were slower than letter comparisons $[F(1,52)=$ $16.162, p<.001, M S e=12944.872$ ], but the letter comparison advantage was much more evident on simultaneous presentation $[F(1,52)=14.815, p<.001$, $M S \mathrm{e}=12944.872]$. Also, simultaneous stimulus comparison was slower than sequential $[F(1,52)=31.599$, $p<.001, M S \mathrm{e}=12944.872]$.

\section{DISCUSSION}

One of the purposes of the research reported here was to investigate the size of the same-different discrepancy in a multistimulus matching task. The findings of this experiment verified those of previous studies that "same" judgments are faster than "different" (e.g., Bamber, 1969; Nickerson, 1965; Pachella \& Miller, 1976). Latencies were about $90 \mathrm{msec}$ faster when the subject compared same stimuli than when comparing different stimuli. Regression analysis showed a considerably higher slope with this task than with the classical Sternberg (1966) memory task. According to additive factor logic (Sternberg, 1969), the slopes of this function should be considered a composite of search and comparison and response choice, because the number of response alternatives is not constant throughout the set size variable. Nevertheless, the difference between slopes for positive and negative responses should not be attributed to response choice, because both types of responses represent the same amount of uncertainty. Rather, the discrepancy could be attributed to some basic difference between the comparison process carried out on both stimulus sets.
Also, as was expected, reaction time increased linearly with stimulus set size, showing that the subject has to exhaustively scan the whole set of items before making a response. The neutralization of the selftermination factor did not affect the speed advantage for "same" responses for simultaneous pairs, thus conflicting with Krueger's data (1984). The self-termination principle proposed by Krueger predicts that discrepancy between "same" and "different" judgments will be reduced with stimuli presented simultaneously. The analysis shows no significant effect of the presentation type $\times$ response type interaction. The same-different disparity and the fast same effect were obtained regardless of whether subjects compared stimuli presented in a continuous or in a discrete manner. That is, the self-termination scan did not explain all the differences found between "same" and "different" judgments. By contrast, the discrepancy between "same" and "different" responses increased in the larger sets. When the number of common elements between both sets was larger, the time to compare identical items decreased with respect to "different" comparisons. This result can be produced by a priming factor, as Proctor (1981) formulated, and it conflicts with Krueger's data (1984, Experiment 1). Proctor proposed that the priming principle produces facilitation when the prime is physically identical to a subsequent stimulus $(1981$, p. 294). In the experiment reported here, the subject had to respond to the number of physically identical items, and the phenomenon of responding faster to same stimuli may be due to facilitation in the comparison process, not only to the encoding of repeated stimuli as Proctor concluded. Furthermore, neither the method of presentation nor the type of stimulus reversed the "same" judgments advantage.

\section{REFERENCES}

Algarabel, S., \& Sanmartin, J. (1985). Baspal: Descripción de la base computarizada de palabras de la Universidad de Valencia. Psicológica, 6, 189-200.

BAMBER, D. (1969). Reaction times and error rates for "same"-" "different" judgments of multidimensional stimuli. Perception \& Psychophysics, 6, 169-174.

Juilland, A., \& Chang-Rodriguez, E. (1964). Frequency dictionary of Spanish words. London: Mouton.

Krueger, L. E. (1983). Probing Proctor's priming principle: The effect of simultaneous and sequential presentation on same-different judgments. Journal of Experimental Psychology: Learning, Memory, \& Cognition, 9, 511-523.

KRUEGER, L. E. (1984). Self-termination in same-different judgments: Multiletter comparison with simultaneous and sequential presentation. Journal of Experimental Psychology: Learning, Memory, \& Cognition, 10, 271-284.

NiCKERSON, R. S. (1965). Response times for “"same"-“different" judgments. Perceptual \& Motor Skills, 20, 15-18.

Pachella, R. G., \& Miller, J. O. (1976). Stimulus probability and same-different classification. Perception \& Psychophysics, 19, 29-34.

Proctor, R. W. (1981). A unified theory for matching-task phenomena. Psychological Review, 88, 291-326.

REED, S. K. (1973). Psychological processes in pattern recognition. New York: Academic Press.

Silverman, W. P. (1974). The effect of irrelevant surround on speeded visual discriminations varying in complexity. Perception \& Psychophysics, 15, 320-324.

STERNBERG, S. (1966). High-speed scanning in human memory. Science, $153,652-654$.

STERNBERG, S. (1969). The discovery of processing stages: Extensions of Donders' method. Acta Psychologica, 30, 276-315.

STERNBerg, S. (1975). Memory scanning: New findings and current controversies. Quarterly Journal of Experimental Psychology, 27, $1-32$.

(Manuscript received for publication August 3, 1987.) 